\title{
Smart Station: Um Sistema Pervasivo de Notificação em Paradas de Ônibus para Pessoas com Deficiência Visual
}

\author{
Lucas Agostini ${ }^{1}$, Alex Braga ${ }^{1}$, Julio Machado ${ }^{1}$, Alysson Nogueira ${ }^{1}$, Tatiana \\ Tavares $^{2}$, Adenauer Yamin ${ }^{4}$
}

${ }^{1}$ CDTec - Centro de Desenvolvimento Tecnológico - Universidade Federal de Pelotas (UFPel)

\author{
Pelotas - RS - Brasil \\ \{lbagostini, abbraga, jmdsneto, anrodrigues, \\ tati, adenauer\} @inf.ufpel.edu.br
}

\begin{abstract}
Vision is one of the most important channels for the individual's relationship with the world. Also vision is our main "guidance system". Visual impairment people do not have this "guidance system" as their main orientation. A natural form for adaptation is to take advantage of other senses, as hearing and touch, to provide an independent way to accomplish daily activities. An example is the mobility, especially for accessing urban public transportation. Thus, we propose a solution for notification at bus stops based on pervasive computing, using microprocessors and speakers, centered in visual impairment people.
\end{abstract}

Resumo. A visão é um dos canais mais importantes de relacionamento do indivíduo com o mundo, sendo nosso principal "sistema-guia" de orientação. As pessoas com deficiência visual não possuem esse "sistema-guia" como sua orientação principal. Uma forma de adaptação natural é se benificiar de sentidos alternativos, como audição e tato, para oferecer uma forma independente de realização das atividades do cotidiano. Um exemplo é a mobilidade, especialmente no acesso ao transporte público urbano. Dessa forma, propõe-se uma solução para notificação em paradas de ônibus baseada em computação ubíqua, usando microprocessadores e caixas de som, voltado a pessoas com deficiência visual.

\section{Introdução}

Segundo Garlan et al (2002) o recurso mais precioso em um sistema computacional já não é mais o processador, memória, disco ou rede, mas sim o recurso limitado da atenção humana. A atenção humana é um recurso escasso pois o usuário está muitas vezes focado em atividades do seu cotidiano como andar, dirigir ou falar enquanto acessa algum sistema computacional. Quando o sistema computacional não compete pela atenção do usuário o qual pode disfrutar da informação de forma transparente temos então um ambiente genuinamente pervasivo, ou um ambiente pervasivo distraction-free.

Nas atividades do nosso cotidiano que podem ser facilitadas por ambientes dessa natureza, destacamos o transporte público. Além disso, o transporte público é uma condição necessária para a concretização das relações econômicas e sociais mais

Proceedings of the SBCUP'2016 (Julho 2016) 
desafiadoras da nossa Sociedade e um dos desafios para promoção da mobilidade urbana. Para Vasconcellos (2001) existem várias visões da mobilidade, no entanto, essas visões partem do princípio que a mobilidade está relacionada com a capacidade e/ou habilidade de movimentar-se. No entendimento do Ministério das Cidades (2004): " "a mobilidade está vinculada à qualidade de vida dos locais onde as pessoas moram e para onde se deslocam, devendo estar articulada com o plano de desenvolvimento da cidade e com a democratização dos espaços públicos conferindo prioridades às pessoas e não a veículos". Assim, um plano de desenvolvimento urbano para mobilidade deve abordar aspectos que envolvam atributos de qualidade voltados à sustentabilidade, tais como: (i) minimizar o consumo de espaço e recursos naturais; (ii) racionalizar e gerenciar eficientemente os fluxos urbanos; (iii) proteger a saúde da população urbana; (iv) assegurar igualdade de acesso a recursos e serviços; (v) manter a diversidade social e cultural.

Dentre os atributos supracitados, destacamos a igualdade de acesso a recursos e serviços. No Brasil, o ônibus é o veículo mais utilizado para o transporte público de passageiros, no entanto, a acessibilidade ainda é muito restrita no transporte público, especialmente, para os usuários cegos ou de baixa visão. Quando estas pessoas precisam utilizar o transporte público, elas enfrentam grandes dificuldades, que, segundo depoimentos, são ocasionadas devido a falta de dispositivos adaptados nas paradas de ônibus. Segundo Vasconcellos (2001) essa deficiência do serviço de transporte público muita vezes coloca seus usuários em situação de desconforto e sem autonomia para realizar sua atividade de movimentação urbana como qualquer outro passageiro. Segundo Henry (2014) um dos pontos principais a acessibilidade é o design de produtos capazes de tornar mais autônomas ou independentes pessoas que possuem algum tipo de deficiência, seja de modo direto (sem ajuda de ninguém) ou indireto (com a ajuda de alguém ou alguma coisa/dispositivo). Nesse sentido uma solução pervasiva que permita a igualdade de acesso a recursos e serviços no transporte urbano pode contribuir efetivamente para promoção da acessibilidade.

Neste artigo é apresentado o Smart Station, um sistema pervasivo de notificação capaz de alertar os usuários sobre o status de uma parada de ônibus utilizando avisos ou mensagens sonoras. Como contribuições da proposta, este sistema utiliza um solução de baixo custo e baixo consumo energético capaz de comunicar informações imediatas sobre os ônibus de uma parada. Nossa intenção é, então, oportunizar maior independência aos usuários com deficiência visual no usufruto do serviço de transporte coletivo.

\section{Trabalhos Relacionados}

O aplicativo CitaMobbi Acessibilidade foi desenvolvido por um usuário de ônibus cego e tem como finalidade avisar em tempo real informações para embarque e desembarque de passageiros. Este aplicativo é uma extensão do CitaMobbi, aplicativo mobile que oferece informações em tempo real para transporte público com ônibus em 15 cidades brasileiras. O CitaMobbi Acessibilidade utiliza mensagens de voz e sistema de vibração do celular. Luiz Eduardo Porto, desenvolvedor da versão acessível,

Proceedings of the SBCUP'2016 (Julho 2016) 
acrescenta: "De casa já é possível acionar o aplicativo, programando até a parada final da viagem. E não precisa levar o celular na mão, virando alvo de ladrões, pois dá para perceber ele vibrando dentro do bolso do casaco quando o onibus está chegando" (Uehara, 2015).

Em (Willis, 2005) é apresentada uma solução que utiliza um sistema de grade RFID, como solução para o problema de acessibilidade de alunos cegos. As RFIDs seriam programadas com informações sobre o ambiente ao seu redor, permitindo assim que não seja necessário um sistema centralizado com os dados armazenados ou uma rede wireless para troca de informação. Esse sistema em grade de RFID permitirá também avanços na robótica para o conhecimento preciso de lugares. Para utilização desse sistema, cada usuário levará em seu sapato um leitor de RFID que se comunicará com o usuário pelo celular através de conexão Bluetooth. Para dar assistência a locomoção, o usuário usa um cinto que emite sinais sonoros e vibra, indicando se o mesmo está indo na direção desejada.

De acordo com Scavin (2016) para atender a lei de mobilidade urbana $12.587 / 12$ uma empresa mineira criou um sistema para disponibilizar e equipar ónibus para a maior acessibilidade ao seus usuários cegos. Ao usuário coube adquirir e portar um controle remoto com o sistema embarcado. Essa empresa atua nas cidades de Jáu/SP Araucária/PR e Limeira/SP. No interior de São Paulo foi idealizado pelo grupo criar BUSALERT que tem como o objetivo um sistema que informa a distância, tempo estimado de chegada e o número de paradas até o ponto solicitado pelo usuário. $\mathrm{O}$ diferencial para a melhor acessibilidade é que o sistema funciona em dispositivos móveis, tanto por texto como por áudio, neste último caso, facilitando o manuseio ao deficiênte visual.

$\mathrm{Na}$ mesma linha de desenvolvimento de software para acessibilidade, Bianchi (2014) apresenta o desenvolvimento de um sistema móvel para o controle de transporte coletivo, com o fim de auxiliar a utilização deste por pessoas com deficiência visual. O funcionamento do sistema conta com recursos disponíveis na plataforma Android: como a utilização de GPS, conectividade entre dispositivos e o servidor de dados, sintetização de voz e mapas da plataforma. A execução do sistema é basicamente um aplicativo localizador, em que o aplicativo cliente envia e recebe informações do servidor, executando suas funcionalidades de forma a auxiliar os usuários no transporte coletivo com ou sem deficiência visual. Em síntese o sistema desenvolvido permite que o usuário saiba quando está se aproximando do seu destino através de um aviso sonoro e também indicia o ponto de parada mais próximo além de permitir o recurso de acessibilidade por reconhecimento de voz para que o deficiente visual possa ativar e interagir com o sistema. O intuito do sistema desenvolvido é facilitar o acesso e a autonomia para locomoção dos portadores de deficiência visual.

A Tab. 1 apresenta um resumo dos trabalhos supracitados em função dos aspectos: forma de interação, tipo de informação e dispositivos usuais ou dedicados (hardware específico). Em dispositivos distinguimos os dispositivos ligados ao 
oferecimento da infraestrutura e os necessários para que o usuário tenha acesso ao serviço ou aplicação.

Tabela 1. Comparativo das Soluções Correlatas.

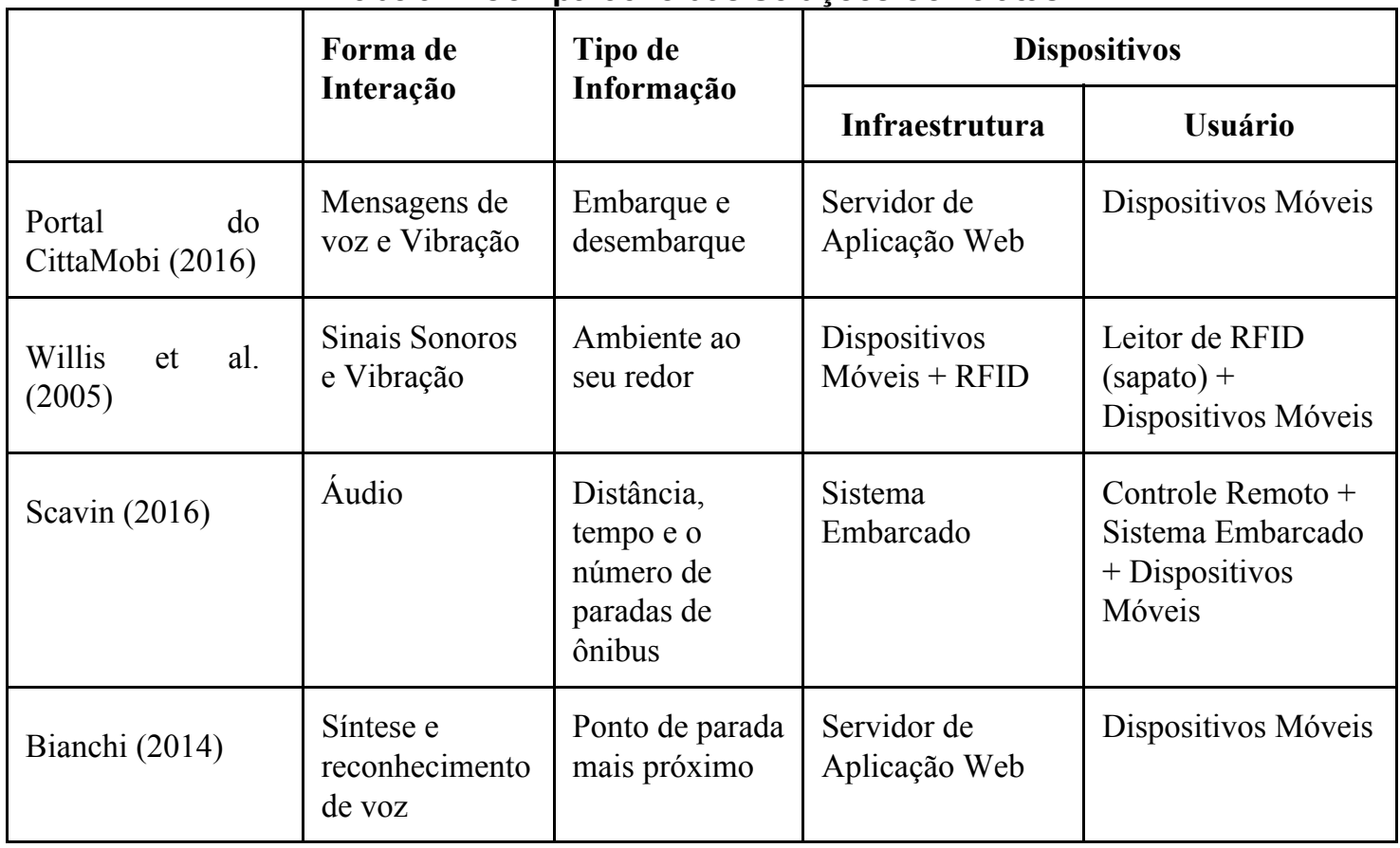

\section{Smart Station: Da concepção ao Desenvolvimento}

Segundo Rocha et. al (2013) sistemas de apoio ao cidadão são de considerável importância. Nesse cenário, a computação ubíqua e ciente de contexto, que se caracteriza pela presença de dispositivos portáteis, aparece como peça fundamental. Nogueira et. al (2012) acrescenta que visando a melhoria desse cenário, o conceito de computação pervasiva tem sido apontado como promissor, pela integração da computação com o cotidiano focada na melhoria da qualidade de vida, saúde pessoal e capacitação de usuário. Aliado a isso, ter em mente o requisito de não colocar na responsabilidade dos usuários usar ou carregar ou iniciar um aplicativo ou dispositivo para acesso ao serviço ou informação nos leva à concepção do Smart Station.

O Smart Station é focado no acesso do usuário com deficiência visual ao transporte público. Diferentemente de soluções baseadas em dispositivos móveis (Portal do CittaMobi (2016), Bianchi (2014)), nosso intuito é prover uma solução que não necessita de gatilho ou dispositivo para sua ativação. A ideia é bastante intuitiva: o usuário que está na parada do ônibus escuta áudio que identifica o ônibus que se aproxima da zona de embarque, ou o usuário que está dentro do ônibus escuta o áudio que identifica a próxima parada. Para tanto, dotamos o ônibus e a parada de microprocessadores ESP8266 e comunicação Wi-Fi conforme ilustrado na Fig.01.

\subsection{Metodologia de Desenvolvimento}

Como dito anteriormente a abordagem "distraction free" imprime um ritmo de desenvolvimento focado no usuário. Este fato também está presente no conceito de 
experiência de usuário (do inglês: "user experience ou $U X$ ") e foi aplicado neste trabalho como uma forma mais ampla de analisar a relação usuário-dispositivo-sistema através da compreensão da interação na perspectiva do usuário. Arhippainen (2003) caracteriza $U X$ pela experiência que o usuário obtém quando interage com um produto. Segundo o autor a $U X$ é definida como as emoções e expectativas do usuário e sua relação com outras pessoas e com o contexto de uso, onde destacam-se aspectos afetivos e experiências pessoais.

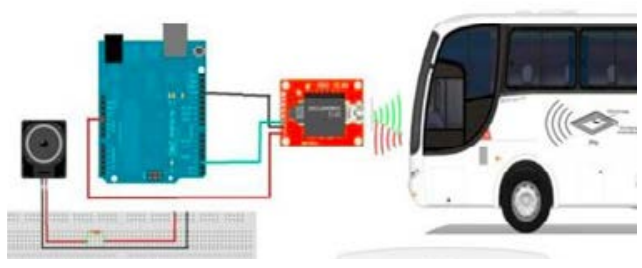

(a)

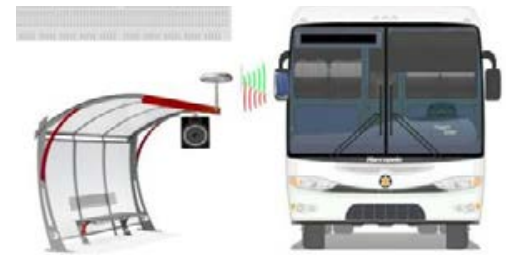

(b)

Figura 1. Visão Geral da Solução.

A Fig.2 apresenta as principais atividades da metodologia de desenvolvimento utilizada no Smart Station. A definição do problema compreende o entendimento do cenário de uso e de soluções correlatas. E, então, é necessário definir o público-alvo através da identificação e descrição das personas. O refinamento dos requisitos nos permite compreender as funcionalidades que neste caso contou a participação dos usuários reais. O design da interação consiste na elaboração de um modelo de execução do sistema como também os protótipos necessários à sua validação. Por fím, o produto final é avaliado. Para a avaliação da interface de áudio utilizamos o sistema MOS (Mean Opinion Score) que dita regras de como avaliar um arquivo de áudio (ou video) através da percepção dos usuários.

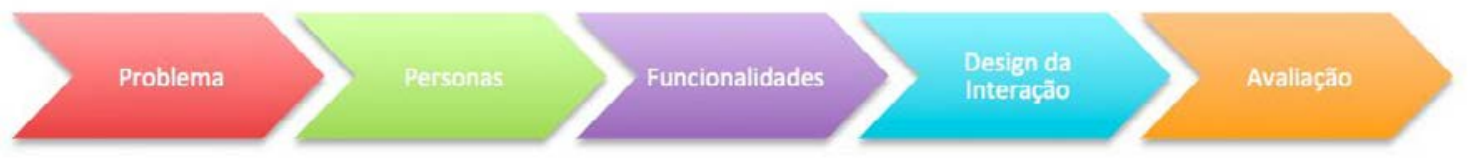

Figura 2. Metodologia de Desenvolvimento Baseada em UX.

\subsection{Personas do Smart Station}

A definição e descrição das personas foi essencial no entedimento da dinâmica de uso do sistema, especialmente, na compreensão da percepção dos elementos físicos responsáveis pela prover a ubiquidade da solução. Nesta etapa foi delineado dois perfis de usuário como principais personas desejáveis:

- Pessoa com deficiência visual que deseja utilizar o transporte público.

- Pessoa que não sabe ler, e que deseja saber o ônibus que está passando.

O entendimento da persona - pessoa com deficiência visual - necessitou de uma aproximação com público-alvo para que pudessemos entender melhor a situação atual e projetar uma solução para a situação desejada (com uso do sistema proposto). Dessa forma, os usuários reais do sistema participaram ativamente do processo de 
desenvolvimento, desde a etapa de entendimento do problema até a etapa de avaliação do produto final. Na Fig.3 (a) é ilustrado um storyboard que representa situações do cotidiano de pessoas com deficiência visual que utilizam transporte público, onde observa-se a total dependência de uma terceira pessoa (motorista ou passageiro) para que a informação de qual ônibus esteja acessível para esses usuários. Já a Fig.3 (b) representa o cenário desejado, onde uma solução pervasiva integrada ao ônibus e a parada de ônibus é capaz de notificar ao usuário com deficiência visual qual o ônibus está chegando na parada.

É interessante destacar, que na análise de requisitos foram levantadas outras funcionalidades como tempo de espera ou frequência de parada, no entando, na reúnião com os usuário a funcionalidade desejada foi: "Eu quero saber qual o ônibus está se aproximando da parada!".

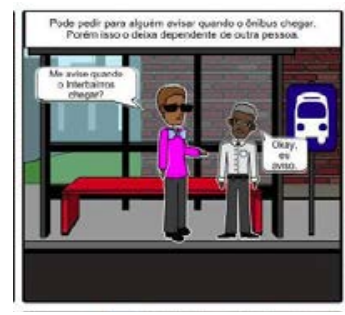

(a)

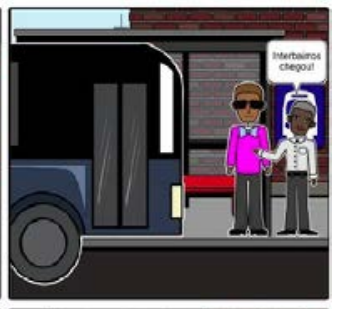

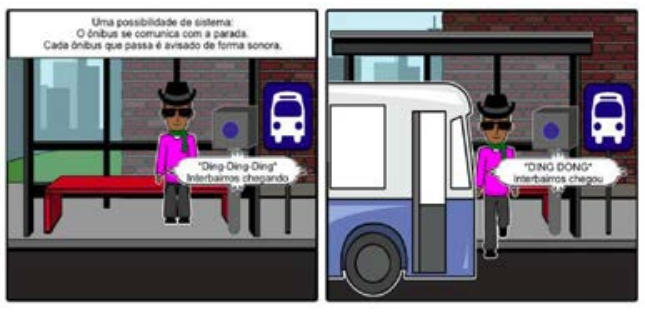

(b)

Figura 3. Storyboard com detalhamento das personas.

\subsection{Smart Station: Tecnologias Utilizadas e Prototipação}

A implementação do Smart Station foi projetada a partir de uma arquitetura estilo cliente-servidor conforme ilustra a Fig.4. O módulo servidor - Server - pode ser detalhado em quatros componentes: AudioFile, Receiver, IPGenerator e Sender. O módulo cliente - Client - possui três componentes: AudioFile, Receiver e Sender. A conexão entre os módulos Server e Client é estabelecida via UDP.

Os módulos servidor e cliente são executados em microprocessadores ESP8266. O microprocessador ESP8266 (Espressif Systems, 2015) possui uma interface de comunicação USB e um módulo de comunicação Wi-Fi, e com este módulo ela consegue fazer comunicação ad-hoc (sem infraestrutura adicional). A comunicação entre duas placas é feita através da criação de um servidor (com uma placa) e um cliente (com a outra placa). Quando elas se aproximam (segundo o datasheet a distância é de $50 \mathrm{~m}$ ) a conexão entre elas é realizada e as informações trocadas. As informações trocadas pelas placas identificam os áudios, ou seja, a placa que esta no ônibus diz a linha do ônibus na qual ela se encontra e a placa que está na parada informa ao ônibus sua identificação.

$\mathrm{Na}$ Fig. 4 temos um diagrama de componentes que ilustra a arquitetura principal do Smart Station. O componente AudioFile designa os arquivos de áudio, cada parada de ônibus tem um arquivo de áudio com seu nome, e cada ônibus possui um arquivo com o nome da linha. O componente Sender é responsável por enviar o AudioFile para o outro ponto da conexão. O componente Receiver é responsável por enviar o áudio 
que está na ESP8266 via conexão sem fio. O componente IPGenerator fica na parte do servidor e é responsável por determinar IPs para os clientes. Os componentes AudioFile, Receiver, IPGenerator e Sender descrevem o servidor. Os componentes AudioFile, Receiver e Sender descrevem o cliente. Com isso, os ônibus possuem uma ESP8266 que funciona como servidor. Enquanto nas paradas dos onibus temos outra ESP8266 funcionando como cliente.

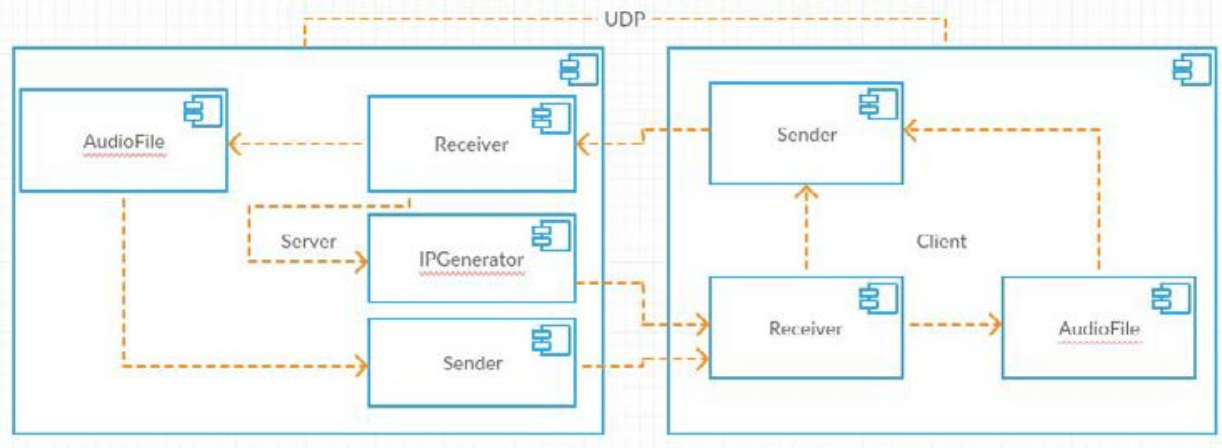

Figura 4. Arquitetura do Smart Station.

Os primeiros testes realizados com protótipos funcionais foram essenciais para conhecer as fronteiras da tecnologia adotada. A primeira preocupação foi entender a relação entre tempo de resposta e tempo de reação dos usuários. Lembrando que a intenção é oferecer um sistema de notificação que além de informar aos usuários qual o ônibus que se aproxima ofereça tempo para que o usuário possa tomar a decisão de solicitar ou não a parada do veículo.

Para execução dessa primeira bateria de testes foi utilizada: (i) para representar o ônibus: a placa conectada a entrada de USB de um veículo de passeio; (ii) para representar a parada: uma placa conectada a um notebook em uma parada de ônibus. Através desses testes foi constatado que: (i) o tempo médio de resposta do sistema é cerca de 3,5 segundos; (ii) a distância de conexão sem o uso de antenas externas e' de aproximadamente 40 metros; (iii) a velocidade de deslocamento do veículo não interfere no tempo de resposta.

\subsection{Avaliação}

Como dito anteriormente o envolvimento com usuários reais é uma característica deste projeto. Para avaliação dos protótipos foi utilizada a metodologia MOS (Rec, I. T. U. T., 2006) procurando identificar clareza, velocidade, volume e o tempo de resposta do sistema. Na escala de avaliação percebida sugerida pela metodologia MOS são apresentados para os voluntários seis opções para medição do grau de satisfação. Para realização dos testes para avaliar clareza, velocidade e volume foram envolvidos 23 voluntários. Para realização dos testes de tempo de resposta foram envolvidos 06 voluntários.

O resultado referente a clareza do áudio percebido pelos voluntários ouvintes obteve uma classificação unânime de perfeito. A Fig.5 (a) denota a avaliação de velocidade e sua relação com a compreensão dos voluntários ouvintes. Podemos

Proceedings of the SBCUP'2016 (Julho 2016) 
observar que a maioria ( $>75 \%)$ atribui conceito perfeito. Foram testados nomes de linhas simples (por exemplo: Pycrespo) e nomes compostos (por exemplo: Santa Terezinha).

A Fig.5 (b) mostra a avaliação do volume a qual também obteve maioria de respostas relacionadas ao conceito perfeito. Esse primeiro conjunto de testes foi realizado em uma reunião do grupo de cegos da Escola Luís Braille. Para se aproximar do contexto real optou-se por fazer uma avaliação coletiva e sem solicitar silência na sala, procurando assim garantir algum ruído. A distância máxima de um voluntário ao ponto de emissão do áudio foi de aproximadamente 5 metros e a distância mínima 1 .

Adicionalmente foi avaliado o tempo de resposta de seis usuários em ambiente real, isto é, na parada do ônibus e os resultados obtidos podem ser visualizados na Fig.5 (c). Após o teste coletivo (Fig.5 (a) e (b)), convidamos usuários voluntários para simular o ambiente da parada de ônibus e cronometramos o tempo de tomada de decisão de cada usuário. Entende-se por tomada de decisão fazer sinal para que o ônibus possar parar. Percebemos que o tempo médio foi de 0,6 segundos e que não ultrapassamos 1,2 segundos.

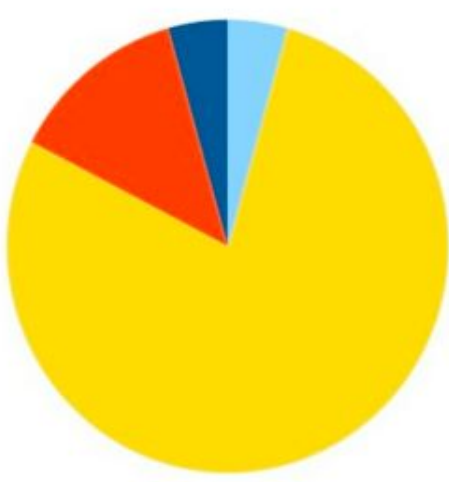

(a)

$$
\begin{aligned}
& \text { = Muito Rapido } \\
& \text { = Rápido } \\
& \text { = Perteito } \\
& \text { = Lento } \\
& \text { = Muito Lento } \\
& \text { = Abstençalo }
\end{aligned}
$$

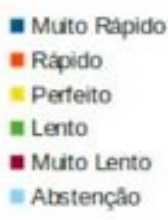

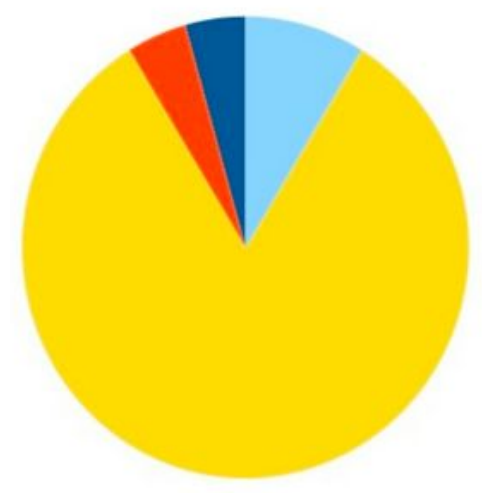

(b)

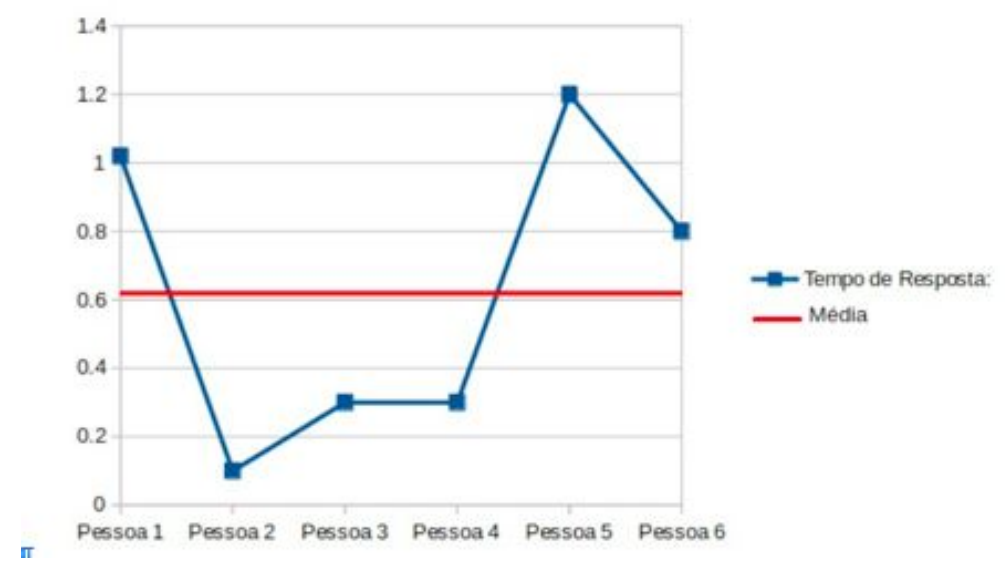

(c)

Figura 5. Resultados. 


\section{Consideraçõe Finais}

Este trabalho apresentou uma experiência de desenvolvimento de uma solução pautada em dois conceitos desafiadores e complementares: tecnologia assistiva e computação pervasiva. Enquanto para o desenvolvimento voltado à tecnologia assistiva a autonomia é uma premissa, para a computação pervasiva exigir menos comprometimento da atenção do usuário, ou seja, atribuir mais autonomia, é mais.

Dentro dessa perspectiva, a Tab.2 apresenta a análise do Smart Station segundo os mesmos aspectos com os quais analisamos os trabalhos relacionados (vide Tab.1). A diferença essencial do Smart Station em relação aos demais é não necessitar que o usuário final vista, use ou acesse nenhum dispositivo ou aplicativo. Essa característica é fundamental para a autonomia percebida pelos usuários finais segundo a perspectiva de tecnologia assistiva. A mesma característica é o que torna a solução distraction-free, em outras palavras, genuinamente pervasiva uma vez que não implica na adição de nenhuma atividade de gatilho ou manipulação de aplicativo ou dispositivo por parte do usuário no ambiente de execução.

Tabela 2. Análise da Solução Smart Station.

\begin{tabular}{|l|l|l|l|l|}
\hline \multirow{3}{*}{$\begin{array}{l}\text { Smart } \\
\text { Station }\end{array}$} & $\begin{array}{l}\text { Forma de } \\
\text { Interação }\end{array}$ & $\begin{array}{l}\text { Tipo de } \\
\text { Informação }\end{array}$ & \multicolumn{2}{|c|}{ Dispositivos } \\
\cline { 2 - 5 } & $\begin{array}{l}\text { Áudio (mensagens } \\
\text { sonoras) }\end{array}$ & $\begin{array}{l}\text { Próximo ônibus e } \\
\text { próxima parada }\end{array}$ & $\begin{array}{l}\text { Servidor + Cliente + placas + } \\
\text { auto-falante }\end{array}$ & $\begin{array}{l}\text { Não } \\
\text { necessita }\end{array}$ \\
\hline
\end{tabular}

Dessa forma, o resultado alcançado neste trabalho corrobora com a visão de Vanderheiden (2008), que destaca que o objetivo de uma solução em tecnologia assistiva é habilitar qualquer um, em qualquer lugar, para ser capaz de utilizar os serviços que necessitam em qualquer computador/dispositivo que encontrarem. Objetivo inerente a soluções em computação pervasiva. Portanto, a tecnologia assistiva se beneficia da computação pervasiva no intuito de oferecer serviços cada vez mais transparentes e integrados ao cotidiano dos usuários.

\section{Agradecimentos}

Nosso agradecimento especial aos alunos, professores e colaboradores da Escola Especial Louis Braille pela sua contribuição essencial para realização deste projeto.

\section{Referências}

Henry, Shawn Lawton, Shadi Abou-Zahra, and Judy Brewer. "The role of accessibility in a universal web." Proceedings of the 11th Web for All Conference. ACM, 2014.

Vasconcelos, Eduardo de Alcântara.Transporte Urbano, espaço e Equidade: análise das políticas públicas,São Paulo:Annablume, 2001.

Nogueira, Carolina, Hyggo Almeida, and Angelo Perkusich. "Arcabouço para o Desenvolvimento de Aplicações de Monitoramento Remoto e Auxílio de Pessoas com Doença de Alzheimer." In: Anais do Simpósio Brasileiro de Computação

Proceedings of the SBCUP'2016 (Julho 2016) 
Ubíqua e Pervasiva (SBCUP), Congresso da Sociedade Brasileira de Computação (CSBC). 2012.

Pansanato, Luciano TE, Christiane E. Silva, and Luzia Rodrigues. "Uma Experiência de Inclusão de Estudante Cego na Educação Superior em Computação." XX Workshop sobre Educação em Computação. 2012.

Rocha, A., et al. "YouOnAlert: Um Sistema para Alertar Cidadãos Comuns Acerca de Problemas do Cotidiano das Cidades." In: Anais do V Simpósio Brasileiro de Computação Ubíqua e Pervasiva (SBCUP), Maceió-Brazil, 2013.

Willis, S., \& Helal, S. (2005, October). RFID information grid and wearable computing solution to the problem of wayfinding for the blind user in a campus environment. In IEEE International Symposium on Wearable Computers (ISWC 05).

Bianchi, Evaldo Augusto. "Sistema para controle de frotas do transporte coletivo com acessibilidade para deficientes visuais." (2014) Disponível em http://repositorio.roca.utfpr.edu.br/jspui/handle/1/2002.

Espressif Systems. "ESP8266EX Datasheet Version 4.3." (2015) Disponível em http://www.esp8266.com/wiki/lib/exe/fetch.php?media=0a-esp8266 datasheet en v 4.3.pdf.

Arhippainen, L. Capturing user experience for product design. IRIS26, $26^{\circ}$ Seminário de investigação de sistemas de informação na Escandinávia, Porvoo, Finlândia 2003. Disponível em: http://www.vtt.fi/virtual/adamos/material/arhippa2.pdf

Uehara, Fernanda. App ajuda deficiente visual a andar de ônibus: Desenvolvido por cego, CittaMobi Acessibilidade usa comando de voz para embarque de passageiro. Disponível

em: http://www.diariosp.com.br/noticia/detalhe/85852/app-ajuda-deficiente-visual-a-anda r-de-onibus Data de Publicação: 05/09/2015 Acessado em: 05/03/2016.

Portal do CittaMobi. Disponível em: < $\underline{\text { http://www.cittamobi.com.br/> }}$. Acessado em: 05/03/2016.

Scavasin, Flávio. "Aplicativos para Segurança e Acessibilidade no Transporte Público" In: Caderno de Cidades - Acessibilidade 2016 - Revista Nacional de Reabilitação Edição número 90, 2016.

Bianchi, E. A. (2014). Sistema para controle de frotas do transporte coletivo com acessibilidade para deficientes visuais. (Monografia de Conclusão de Curso).

Rec, I. T. U. T. (2006). P. 800.1, Mean opinion score (MOS) terminology. International Telecommunication Union, Geneva.

D. Garlan, D. P. Siewiorek, A. Smailagic and P. Steenkiste, "Project Aura: toward distraction-free pervasive computing," in IEEE Pervasive Computing, vol. 1, no. 2, pp. 22-31, April-June 2002.

Vanderheiden, Gregg C. "Ubiquitous accessibility, common technology core, and micro assistive technology: Commentary on "computers and people with disabilities"." ACM Transactions on Accessible Computing (TACCESS) 1.2 (2008): 10.

Proceedings of the SBCUP'2016 (Julho 2016) 